\title{
Coulisses
}

Revue de théâtre

8 | Eté 1993

Varia

\section{Le théâtre universitaire : jeux et enjeu}

\section{Robert Germay}

\section{(2) OpenEdition}

Journals

Édition électronique

URL : http://journals.openedition.org/coulisses/2514

DOI : $10.4000 /$ coulisses. 2514

ISSN : 2546-9460

\section{Éditeur}

Presses universitaires de Franche-Comté

\section{Édition imprimée}

Date de publication : 1 juillet 1993

Pagination : $32-39$

ISSN : 1150-594X

\section{Référence électronique}

Robert Germay, «Le théâtre universitaire : jeux et enjeu », Coulisses [En ligne], 8 | Eté 1993, mis en ligne le 15 mars 2019, consulté le 21 octobre 2019. URL : http://journals.openedition.org/coulisses/2514 ; DOI : 10.4000/coulisses. 2514

Ce document a été généré automatiquement le 21 octobre 2019

Coulisses 


\section{Le théâtre universitaire : jeux et enjeu}

\section{Robert Germay}

Cet article est le texte d'une communication donnée à Lisbonne lors du 3ème Congrès mondial de Sociologie du Théâtre (29/10-03/11/1992) 
1 Je voudrais commencer par «me situer » en tant que « théâtreux » universitaire. Je ne suis pas historien - spécialiste du théâtre universitaire mondial, pas plus que sociologue. Je suis un philologue littéraire "happé » progressivement par le théâtre en tant que pratique - l'étude de la théorie est venue par la suite... jusqu'à devenir aujourd'hui objet de mon enseignement à l'Université de Liège : ma connaissance du théâtre a d'abord été empirique, viscérale même. Ayant

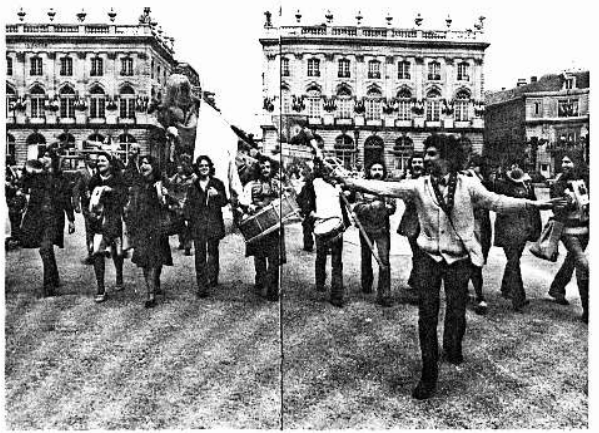
commencé comme étudiant germaniste, (j'ai co-fondé en 1962 un théâtre universitaire de département) et, dirigeant aujourd'hui le Théâtre Universitaire de Liège, j'ai vécu et je vis encore - l'histoire d'un théâtre étudiant en particulier, mais aussi l'histoire du Théâtre Universitaire en général par ma participation à de nombreux festivals et tournées internationales soit avec nos spectacles, soit en observateur/conférencier. C'est à ce titre que je vous parle aujourd'hui.

Aujourd'hui signifie à un moment où le Théâtre Universitaire se repose - pour la $\mathrm{X}^{\text {ème }}$ fois - avec rigueur, parfois avec passion, la grande question de son identité et de son unité. Jacques Lasalle a dit ${ }^{1}$ que " être acteur (...) c'est vivre un déséquilibre, une crise d'identité, une incertitude d'être. Qui suis-je et suis-je seulement? » Je suis tenté d'étendre cette définition à tout le théâtre universitaire même.

Faisons d'abord un peu d'histoire. ${ }^{2}$

3 1. Je n'ai pas fait de recherches sur les origines du théâtre universitaire, mais il est connu qu'on atteste des représentations données dès le Moyen Age par des étudiants de Vilnius (Lituanie), en Pologne et sans doute dans la plupart des grandes universités dès leur création.

2. On s'accorde aussi à situer dans les années 1930 la naissance du théâtre universitaire, disons, moderne. Quelques exemples fameux en France :

1932 : le Théâtre des Etudiants dirigé par Franc Nohain et Jean Delage ;

1933 : création avec Gustave Cohen du Groupe de Théâtre Médiéval de la Sorbonne appelé Les Théophiliens (en raison de leur premier spectacle, Le Miracle de Théophile de Rutebeuf) ;

1936 : création par Roland Barthes et Jacques Weil du Groupe de Théâtre Antique de la Sorbonne. Dans le même temps, se créaient en province des troupes telles Les Comédiens d'Aquitaine (Bordeaux), Les Escholiers de Picardie (Lille), Les Escholiers du Languedoc (Montpellier)... A croire d'ailleurs que la Wallonie est aussi une province française puisqu'au même moment le Cercle de Philosophie et Lettres de l'Université de Liège jouait du Banville, mais aussi des Nativités médiévales ou de l'Euripide... en grec, tandis que le cercle de Philologie germanique jouait en langue originale des auteurs allemands, anglais et flamands, ou hollandais. Des échanges eurent d'ailleurs lieu en 35-36 entre les Liégeois et les Théophiliens de la Sorbonne (pour la petite histoire et par parenthèse, Gustave Cohen était de père français et de mère belge et avait conquis, en 1903, le titre de Docteur en Philosophie et Lettres... à l'Université de Liège !). Tout cela devait d'ailleurs conduire à la très officielle reconnaissance du "Théâtre Universitaire Liégeois » par les autorités académiques en 1941. (Le TULg n'a jamais cessé ses activités 
depuis, au contraire de beaucoup de ses collègues français - ou européens contemporains.)

Ce théâtre universitaire-là (qu'il soit de France, de Belgique, ou d'ailleurs...), était en tout cas le reflet de cette université-là : avec le théâtre, s'il était bien sûr une activité de loisirs, le « para-universitaire » rejoignait vite l'« universitaire » tout court. En liaison étroite avec les matières enseignées et souvent avec les enseignants eux-mêmes, l'activité théâtrale était une sorte d'approfondissement et/ou de complément des "chères études", un hobby tout empreint de la rigueur, sinon de l'austérité, de l'apprentissage scolaire, un terrain d'application de la recherche, des «travaux pratiques » de l'enseignement... Ceci, d'ailleurs, n'enlève rien aux qualités esthétiques de ces productions d'amateurs, qui faisaient montre d'une inventivité certaine sur le plan du théâtre même : l'éclectisme du répertoire - choix de « pièces rares »-, le soin apporté aux costumes, à la diction, à la chorégraphie... On allait parfois jusqu'à composer des musiques originales pour la circonstance (c'était le cas à Liège) et la mise en scène reposait sur une analyse textuelle fouillée qui préfigurait souvent ce qu'on appelle aujourd'hui la dramaturgie. Sans en avoir les moyens, le théâtre universitaire essayait de faire aussi bien, mais dans un autre registre, que le théâtre professionnel, d'une part, et d'autre part, il se distinguait du théâtre amateur non-universitaire par la rigueur intellectuelle de l'analyse de l'œuvre, et, dans certains cas, par le choix de la langue.

Ce théâtre-là était, vu de l'extérieur, conforme à son milieu, et, vu de l'intérieur, parfaitement intégré, voire rassurant. Mais tout en restant l'auxiliaire direct de l'enseignement et de la recherche, le théâtre universitaire, par ses activités publiques, ouvrait une brèche dans une tour d'ivoire où la vie culturelle n'avait sinon guère cours.

5 3. La seconde guerre mondiale, la formidable et terrifiante déchirure qu'elle représente (rupture de la tradition, état de manque de culture, destruction même d'un potentiel humain) va provoquer un bouleversement considérable et irréversible de cette façon de pratiquer le théâtre à l'Université, et, on le verra, le théâtre tout court. Il n'est pas étonnant que ce soit précisément en Allemagne, dès le lendemain de la guerre, que le théâtre universitaire a connu une évolution rapide et profonde.Plus que quiconque, les jeunes Allemands ressentaient le besoin de combler le vide culturel qu'avaient provoqué d'abord les années brunes - comme on les appelle pudiquement - et ensuite la guerre. En rupture de tradition, méfiants envers les aînés, ils allaient, non pas reconstruire le théâtre, mais en construire un autre à leur façon et à leur image.

C'est ainsi que naît à Erlangen en 1949 le premier grand Festival International de Théâtre Universitaire : il répondait d'une part à un besoin d'ouverture à l'extérieur, de "réconciliation» avec l'Europe et le Monde, de découvertes culturelles, mais aussi d'autre part à une réadéquation des contenus et des formes du théâtre dans un monde si différent. Erlangen fut pendant des années un extraordinaire terrain d'expérimentation, tant pour les « faiseurs » de théâtre que pour les auteurs et même le public, les critiques et les chercheurs. Loin de rester local, le choc que constituait chaque année l'événement théâtral d'Erlangen se répercuta dans tout le pays. On n'en finirait pas de citer les troupes étudiantes, mais aussi les personnalités qui sont passées par Erlangen : d'Adamov à Günter Grass, de Claus Peymann à Enzensberger ou John Arden. Car les « Semaines internationales de Théâtre étudiant » étaient bien plus qu'un simple festival, qu'une simple vitrine de pratiques théâtrales: c'était aussi un lieu privilégié de confrontations d'idées et de théories. La presse, très attentive à ce qui se passait là est unanime : au Festival d'Erlangen, le théâtre étudiant a, dans les années 50, 
marqué d'une empreinte profonde le théâtre allemand dans tous les domaines. C'est d'ailleurs aussi à Erlangen que fut créée en 1954 la première Union Européenne du Théâtre Universitaire, dont la Fédération Nationale des Théâtres Universitaires (FNTU) française, toute jeune encore - elle avait été reconnue officiellement en 1947 - est l'un des membres fondateurs. Il n'est pas trop audacieux, enfin, de penser que l'influence d'Erlangen n'est pas étrangère à la création en 1963 du fameux festival mondial de Théâtre Universitaire de Nancy, sous la direction de Jack Lang.

C'est, je crois, deux faits marquants des années 50 : le théâtre étudiant, parfaitement amateur, en affirmant haut et clair sa spécificité mais aussi sa qualité par rapport au théâtre professionnel, forçait celui-ci à remettre en question sa propre pratique tant sur le plan du répertoire que sur celui de la dramaturgie, voire même celui du rapport au public. Ce faisant, par ailleurs, - peut-on appeler cela un "effet pervers" ? - le théâtre universitaire allait perdre nombre de ses membres à qui il avait servi de tremplin vers le professionnalisme. Un des exemples français, le plus connu est sans doute celui d'Ariane Mnouchkine qui, en 1959, fondait à la Sorbonne L'Association Théâtrale des Etudiants de Paris dont allait sortir en 1964 le Théâtre du Soleil. Parallèlement, et c'est l'autre fait marquant : le théâtre, par la lumière qu'il attirait de plus en plus sur lui, prenait dans l'Université, côté académique et côté étudiant, une importance de plus en plus marquée. Dans les années 60, le monde étudiant allait bientôt utiliser le théâtre à des fins politiques. L'apparition de plus en plus fréquente des théâtres étudiants de l'Est de l'Europe - Pologne, Tchécoslovaquie, Yougoslavie n'y est sans doute pas non plus étrangère. La période faste qu'a connue par exemple la FNTU française entre 1964 et 1966 - dixit Richard Monod ${ }^{3}$ - elle la doit sans doute au soutien, certes provisoire mais actif, de l'Union des Etudiants de France (UNEF) (par Jean-Jacques Hocquard interposé). De même en Belgique, à Bruxelles par exemple, le MUBEF (Mouvement des étudiants universitaires belges d'expression française) organisait en 1964 un premier festival de théâtre étudiant dans le programme duquel on pouvait lire: "Il s'agit pour les théâtres étudiants de nouer des contacts les plus étroits possibles avec le monde du travail et d'adapter son répertoire en conséquence ». Et en Allemagne aussi le mouvement étudiant a vu longtemps dans le théâtre universitaire un moyen d'agitation sociale. Mais en une sorte de cercle vicieux, la contestation (avec un grand C) a eu raison de cette nouvelle orientation des théâtres universitaires. Le conflit manifeste entre théâtre comme moyen d'expression artistique et théâtre - outil politique conduisait à un éclatement.

En Allemagne d'abord où la fraction la plus radicale du mouvement étudiant réclame à cors et à cris la fin du Festival d'Erlangen, ce qu'elle obtient de fait en 1968. ${ }^{4}$ En France aussi, mais plus tard, jusqu'en 1975, la FNTU qui privilégiait « une orientation très militante du Théâtre Universitaire : théâtre-tract, agit-prop, créations collectives ${ }^{5}$ abandonnera cette position lors de l'Assemblée Générale de Clermont-Ferrand en 1975. Quoi qu'il en soit, pendant les années 50 et 60 , le théâtre étudiant venait de «bousculer» par son dynamisme le théâtre professionnel. Il s'était révélé une pépinière de nouveaux talents dont beaucoup sont aujourd'hui des professionnels célèbres. Il avait enfin considérablement accru son quotidien à l'extérieur et à l'intérieur de celle-ci, son importance: l'institution ne pourrait plus ne pas en tenir compte.

6 4. Et, sans doute, par voie de conséquence, le fait marquant des années 70 restera l'apparition, un peu partout dans les universités d'Europe, d'Instituts de Recherches Théatrales, un peu comme si on voulait rattraper l'avance prise en ce domaine dans le 
monde anglo-saxon. La FNTU demanda même en 1977-78 la création systématique de tels Instituts dans chaque université et "la prise en considération de travaux des troupes universitaires dans certains diplômes universitaires $» .{ }^{6}$ Beaucoup d'institutions ont cédé. Et ce n'était qu'un début : le combat continue.

7 5. Pour clore ce trop bref survol historique, je soulignerai pour les années 80 un fait nouveau: l'incroyable profusion de Festivals internationaux de Théâtre universitaire qu'il m'a été donné de visiter soit avec notre troupe, soit en observateur : j'en compte aujourd'hui 24, pour la plupart bien établis et récurrents, de Vilnius à Manizales en Colombie, de Tampere (SF) à Casablanca, de Montréal à Campinas (Brésil) ou de Liège... à Louvain-La-Neuve. Et je ne compte pas les Festivals des Grandes Ecoles françaises. Cela montre bien, je crois, non seulement la toujours grande vitalité du théâtre étudiant, mais aussi sa volonté d'échanges tant sur le plan humain que sur celui des pratiques théâtrales. Et quand on voit les Institutions universitaires elles-mêmes soutenir et promouvoir ces rencontres, c'est aussi le signe qu'elles y voient le reflet de leurs propres aspirations à s'ouvrir - se vendre? - de plus en plus et sur tous les plans au monde extérieur: la brèche dont je parlais plus haut. Cela montre enfin qu'un changement s'opère, lentement mais sûrement, par rapport à une si longue indifférence du milieu enseignant envers la vie culturelle.

Si cette évolution, brossée ici à grands traits, montre surtout la place qu'a occupée le théâtre selon les époques dans l'université, elle reflète tout autant, corollairement, les grandes mutations qu'a connues l'image même de la vie universitaire: jusqu'à la guerre, université d'une élite, refermée sur elle-même où le théâtre reflétait surtout la vitalité d'une section, d'un département et/ou l'aura de quelques " Maitres »; puis sous les secousses d'une crise générale de société, mise en question de tout un système de rapports entre enseignants et enseignés, et ouverture vers le monde social; enfin, besoin de l'université de s'intégrer aujourd'hui de plus en plus dans le monde sur le plan régional, national et international. Et c'est la création de nouveaux départements (théâtre, communication), c'est la mise en place d'« interfaces » pour plus de liens avec le monde marchand, ce sont Erasmus, Lingua... et les Festivals, "images de marque ». Oui, le théâtre universitaire a toujours peu ou prou été le miroir de la place qu'occupe ou que cherche l'université dans la société.

Bien entendu, je ne suis pas dupe : résumer comme je l'ai fait l'histoire du théâtre en essayant de mettre en évidence des lignes de force c'est courir le risque d'une généralisation réductrice. Car évidemment chaque théâtre universitaire garde une spécificité propre : Liège n'est pas Bruxelles, Dijon n'est pas Besançon, Moscou n'est pas Montréal. De même la Pologne n'est pas l'Italie et l'histoire du théâtre universitaire de ces deux pays mériterait pour chacun un livre entier.

En effet, l'activité théâtrale - non seulement les " produits finis ", mais aussi et d'abord la méthode de travail - en dit souvent long sur le milieu et l'époque où elle se pratique. Et c'est bien pour cela, que malgré une évolution globalement plutôt positive de l'ensemble en ce qui concerne leur place et dans l'université et dans le paysage théâtral, les théâtres universitaires n'en finissent pas de se questionner sur leur identité et sur leur fonction. La prolifération de théâtres universitaires, l'internationalisation des échanges par les tournées et les festivals ont d'une part suscité un désir d'unité, d'organisation, de fédération, et d'autre part, mis en lumière les différences. Et celles-ci ont rendu plus difficile, plus caduque, toute volonté de se rassembler (se ressembler ?).

Le théâtre universitaire n'est en effet pas monolithique. En 1962 déjà, lors d'une 
Assemblée Générale de la FNTU s'ouvrait un grand débat sur la fonction du théâtre universitaire en France: "Le théâtre universitaire est-il une activité de loisirs du milieu étudiant, une illustration des cours de théâtre dispensés dans les universités, l'antichambre du professionnalisme ${ }^{7} .^{7}$ Exactement 30 ans plus tard, un colloque organisé à Liège en février 1992 dans le cadre des $9^{\text {èmes }}$ Rencontres Internationales de Théâtre Universitaire débattait des mêmes questions, cette fois à un niveau international: des participants de 17 pays de trois continents étaient présents à ce colloque (Allemagne, Belgique, Brésil, Québec, Danemark, France, Grande-Bretagne, Irlande, Lituanie, Maroc, Mexique, Pays-Bas, Pologne, Sénégal, Espagne, Finlande, Russie) représentants, certes, de théâtres universitaires, mais aussi d'associations étrangères au mouvement, œuvrant en milieu amateur ou professionnel, tels par exemple : l'AITA (Association Internationale de Théâtre Amateur), l'IIT- belge (section francophone), mais aussi le Théâtre National de Belgique ou le Festival de Cracovie. Les débats, souvent passionnés, ont montré à suffisance combien sont différents les objectifs, les méthodes et les moyens des théâtres universitaires d'une ville, d'une région, d'un pays, d'un continent à l'autre. Mais on s'est accordé assez rapidement sur une définition à trois termes qui semble applicable aux différents pays et qui ressemble fort à celle de la FNTU de 1962 : distinguons

- le théâtre «spontané » : issu de groupes d'étudiants de toute origine ;

- le théâtre « encadré » : pratiqué par des étudiants sous la conduite de professeurs et/ou de professionnels ;

- le théâtre "pré-professionnel » : destiné à former des étudiants en art dramatique et/ou en recherches théâtrales.

\section{Le T.U. de Vilnius en répétition pour Cymbeline}

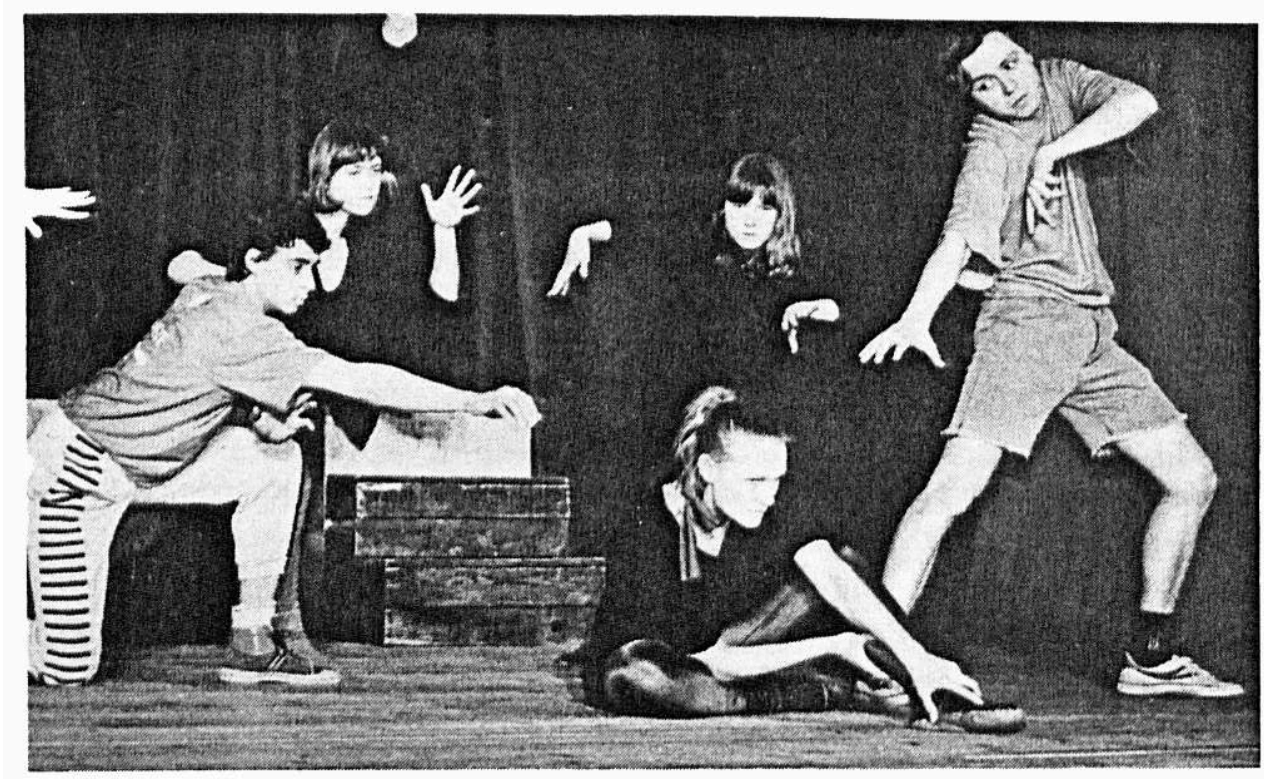

8 Les deux premières catégories existent toujours et on connaît bien les difficultés qu'elles vivent.

La mouvance des étudiants d'abord: c'est une des différences essentielles d'avec le théâtre amateur "ordinaire ». Quoi de plus normal que l'étudiant fasse tout pour que son passage à l'Université soit le plus bref possible, et rares sont ceux qui, une fois entrés dans la vie dite active, continuent leurs activités théâtrales. Les différences dans 
la formation d'abord, les inégalités de talent et de motivation ensuite, sont autant de freins supplémentaires.

Et, plus nouveau ceci : une scolarisation de plus en plus grande des études qui se remarque dans beaucoup d'universités (la multiplication des contrôles réguliers, et la surabondance des matières, entre autres), donne plus de mérite encore à ceux qui continuent à «faire » de la culture plutôt que de la subir, et cela au milieu d'une masse de plus en plus grande de condisciples abrutis par le travail journalier. (Signe des temps certainement : depuis 5 ans, les étudiants liégeois ont réinstauré une annuelle et très sérieuse élection de Miss Université ! Belle alternative ...)

Quant à la catégorie du théâtre "encadré ", aux difficultés citées plus haut, s'ajoute celle de trouver les moyens de transmettre un héritage et une qualité, c'est-à-dire institutionnaliser en quelque sorte, mais tout en préservant la spontanéité : ce type de théâtre, souvent lié intimement à des personnes, généralement bénévoles, fait toujours un peu de la corde raide.

9 La troisième catégorie, elle, le théâtre "pré-professionnel ", si elle existait depuis longtemps dans le monde anglo-saxon, elle a soudainement gagné en importance dans nos pays depuis la floraison nouvelle de cycles de formations théâtrales universitaires. Ici la difficulté majeure est la recherche d'une bonne intégration entre théorie et pratique, opération rendue particulièrement ardue par la carence en moyens financiers et/ou en infrastructures (locaux, matériel technique, personnel...).

Certes, pauvreté n'est pas vice - dirait Grotowski - mais elle n'aide pas le théâtre universitaire à trouver sa place sur le marché professionnel ambiant. Et de développer ainsi, une sorte de complexe (d'infériorité, de supériorité, de frustration ou d'un mélange des trois) que le monde professionnel «marchand» (acteurs, metteurs en scène, producteurs, public même) n'a que trop tendance à renforcer par une attitude souvent indifférente, au mieux condescendante, au pire méprisante, voire hostile.

Les pouvoirs publics eux-mêmes, coincés qu'ils sont dans les cloisonnements ministériels régissant culture ici, enseignement là, accroissent encore ce malaise par leur habituelle politique des vases non communicants. En Belgique francophone, par exemple - c'est presque une " histoire belge ", il n'y a pas dix ans (en 1983), l'Exécutif de la Communauté française (Ph. Moureaux) introduisait dans son budget un nouvel article de «subventions aux théâtres professionnels, semi-professionnels et amateurs, pour l'enfance et la jeunesse, aux théâtres universitaires, aux théâtres professionnels, semi-professionnels et amateurs d'éducation populaire » (9,7 millions de francs belges). ${ }^{8}$ Lors des législatures suivantes, cet article "fourre-tout» a été scindé en deux: subventions aux théâtres pour l'enfance et la jeunesse d'une part, et « subventions aux théâtres universitaires, semi-professionnels, amateurs» d'autre part. En 1991, l'administration s'expliquait ainsi: "Les théâtres universitaires sont spécialement mentionnés dans les budgets de la Communauté française en raison de leur statut hybride, ni tout à fait professionnel ni, sûrement pas, tout à fait amateur $" .^{9}$ La phrase me laisse rêveur : elle révèle plus des états d'âme qu'elle ne définit vraiment le théâtre universitaire et moins encore une politique culturelle.

Le malaise dont je parlais plus haut a, en tout cas, été constamment au centre des débats au récent colloque de Liège. Les discussions sur la définition, la spécificité et l'image du théâtre universitaire dépassent le problème de la terminologie pure.

Si d'un côté, on affirme que la diversité, la spontanéité, le pluralisme constituent la richesse du théâtre étudiant, d'une autre côté, se manifeste la volonté de professionnaliser le théâtre universitaire. 
Se croisent ainsi - s'affrontent même parfois - deux notions :

1. La peur de l'image "amateur", héritière du théâtre étudiant de type "socioculturel », certes dépassée, mais défendue comme la «force et l'origine » de ce qu'est aujourd'hui le théatre universitaire.

2. La difficulté de mettre en exergue pour le valoriser la particularité du théâtre universitaire «pré-professionnel » coincé entre l'amateur et le professionnel «purs et durs ». Le colloque de Liège se savait trop court et trop impromptu pour épuiser un sujet aussi vaste. Rien d'étonnant que les participants aient décidé de se revoir pour tenter de mettre au point une méthodologie qui servirait de base aux travaux du futur Premier Congrès Mondial du Théatre Universitaire. Il devrait - si tout va bien - avoir lieu en octobre 1994 à Liège. Outre l'approfondissement de l'éternelle question de définition, qui pourrait donner lieu à une nouvelle Charte du Théâtre Universitaire, un objectif important du Congrès sera d'améliorer la communication entre les théâtres universitaires à travers le monde, peut-être de mettre sur pied une nouvelle Fédération internationale... Vaste programme!

\section{Le T.U. d'Edinburgh en répétition}

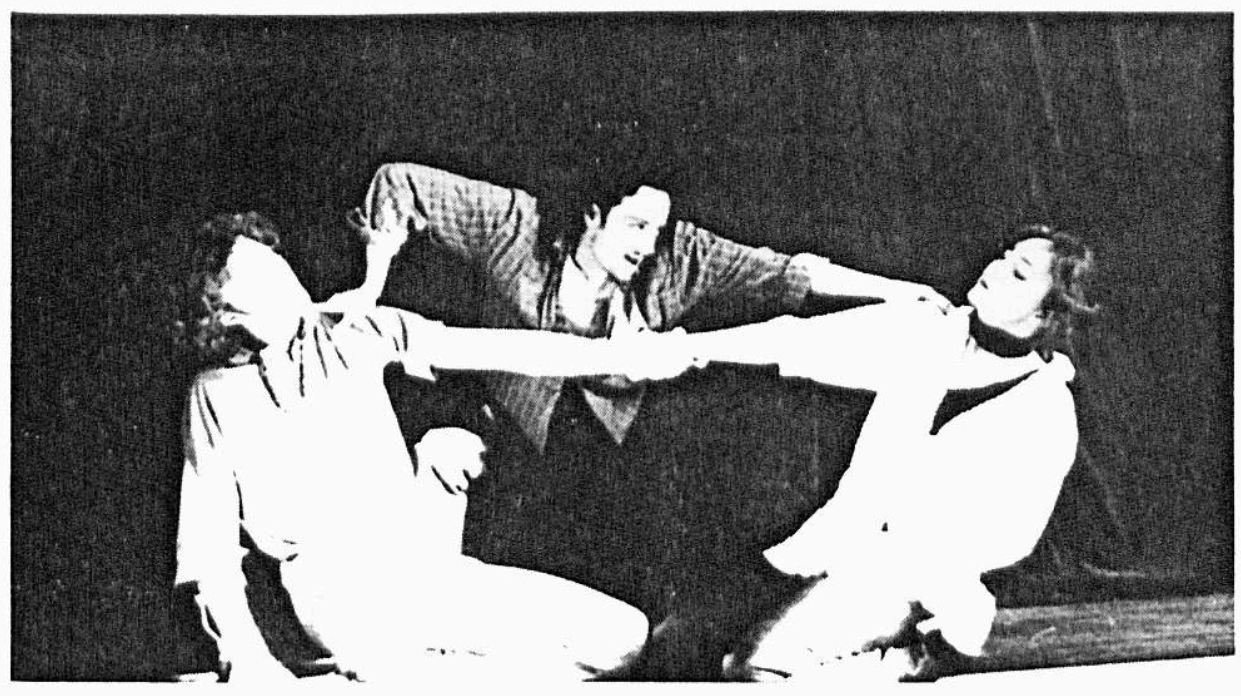

Je m'en voudrais de terminer sur l'impression négative que peut laisser cette évocation des heurs et malheurs du théâtre universitaire d'aujourd'hui. Je rappellerai donc quelques qualités bien connues qui font de la pratique théâtrale à l'université, universellement et de tout temps, un véritable outil de formation et d'apprentissage :

- apprendre un langage : traditionnellement, le drame - et par voie de conséquence, le théâtre - a été rangé dans la catégorie littérature (et ceci oriente souvent le choix du répertoire). On sait qu'aujourd'hui les gens de théâtre ont accentué, renforcé la part du «spectaculaire». Mais le texte dramatique n'en reste pas moins une des composantes essentielles du spectacle, que l'on doit aussi aborder « à la table ». L'analyse littéraire d'un texte dramatique et son analyse en vue de sa représentation ne s'excluent pas : elles se complètent.

- apprendre à être: qui contestera l'importance de la diction, de la pose de la voix, du maintien du corps, du rapport à l'autre dans toute vie d'homme? Qui niera l'apport de la créativité dans le développement intellectuel et culturel de l'individu? Qui mesurera l'enrichissement que représente sur le plan personnel le travail en équipe? 
- apprendre à s'organiser : tout cet acquis dont je viens de parler, il faut encore trouver le courage de le « diffuser » hors du cercle de famille : affronter le public, se confronter à des confrères, et dès lors, organiser le temps de l'étude et le temps du travail « en théâtre ».

11 Bref, la pratique théâtrale à l'université est une autre manière de vivre sa vie d'étudiant et sans doute le lieu de l'université où l'étudiant a le plus droit à l'expression : la culture et l'éducation par la base et non par le sommet de la pyramide.

Outil de formation pour l'étudiant, certes, mais aussi outil de recherche pour le théâtre lui-même. Parce qu'il est libre de préoccupations lucratives, le théâtre universitaire peut s'offrir le temps, et même le nombre, pour prendre des risques - choix du contenu, et choix des formes - et tenter des expériences que d'autres ne peuvent pas souvent se permettre. Comme a dit Mnouchkine : « ... se plonger en pleine ignorance, en toute innocence, à la recherche du théâtre (...). C'est peut-être cela être professionnel, ce n'est pas savoir, c'est savoir chercher ${ }^{10}{ }^{10}$ Le théâtre universitaire a donné mille preuves qu'il sait le faire.

Vaste programme, disais-je, mais l'enjeu est de taille et le jeu en vaut la chandelle. Je laisse le mot de la fin à Roger Deldime : «Les arts [le théâtre], en tant que langage, sont de plus en plus importants dans notre société matérialisée à outrance. La formation artistique [théâtrale] peut être une réponse humaniste à cette dérive matérialiste ${ }^{11}$

Le Jardin des Délices, spectacle du T.U. de Franche-Comté, 1992

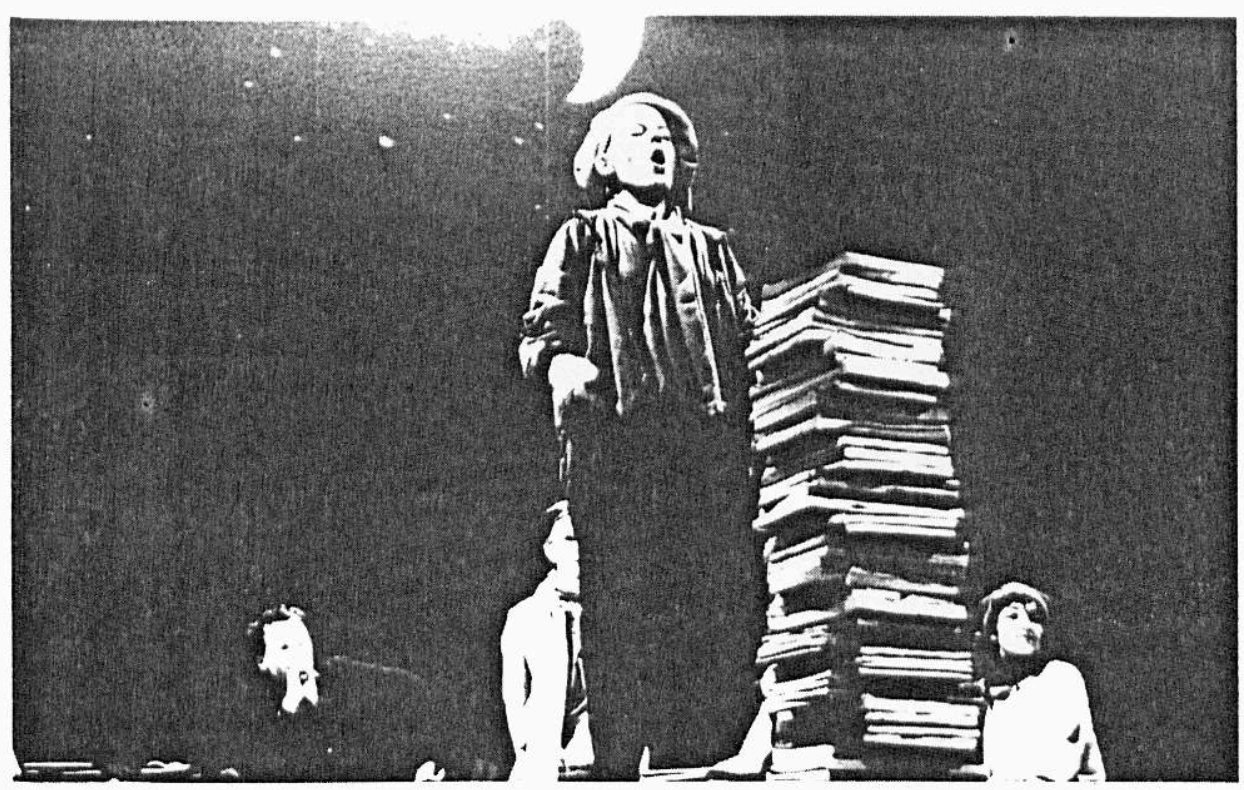

\section{NOTES}

1. Lasalle, Jacques : "Un vivier», in: Théâtre/Public, Hors-série 5 "Théâtre universitaire : quel enjeu ?», Dossier présenté par la FNTU, éd. Théâtre de Gennevilliers, 1984, p. 26. 
2. Dans le même numéro spécial de Théâtre/Public, Marcel Freydefont, alors Président de la FNTU, donne un aperçu historique très utile de l'évolution du théâtre universitaire français de 1932 à 1984, p. 8-12.

Pour ce qui est de Liège, voir: Wathelet, Jeanne/Duysinck, François, «Le Théâtre Universitaire Liégeois ", in La vie wallonne, Liège, Tome 54 (1980), p. 144-153.

Pour Erlangen et l'Allemagne, voir : Hübner, Marlies, Internationales Studententheater in Erlangen, 1946-1968, Erlangen, 1989, 207 p.

3. Théâtre/Public, Hors-série 5, p. 14.

4. Notons que le Théâtre Universitaire d'Erlangen vient de relancer en 1991 un nouveau Festival International qui a vu cette année sa deuxième édition.

5. Ibidem, p. 11.

6. Ibidem.

7. Ibidem.

8. Espace Synthèse, Bruxelles, Ministère de la Culture et des Affaires sociales de la Communauté française, Bibliothèque d'information, $n^{\circ}$ 15/1991, p. 61.

9. Ibidem.

10. Mnouchkine, Ariane, «Savoir chercher ", Théâtre/Public, Hors-série, 5, p. 23.

11. In Le Soir, Bruxelles, 27.8.92, p. 2.

\section{AUTEUR}

ROBERT GERMAY

Université de Liège 